\title{
On the Possible Presence of Ice on the Moon ${ }^{1}$
}

\author{
Kenneth Watson, Bruce Murray, and Harrison Brown \\ Division of Geological Sciences \\ California Institute of Technology \\ Pasadena, California
}

It is generally presumed that gases of low molecular weight escape very rapidly from the moon. As a consequence, it has been assumed that volatile substances, such as water, which possess short relaxation times for escape, do not exist there. Urey [1952] recognized that there may be depressions in which the sun never shines, and in which some condensed volatile substances could be present, but he concluded that no solid or liquid water could exist on the moon for more than very short periods of time. Kuiper [1952], following a suggestion by Herzberg, stated that $\mathrm{SO}_{2}$ molecules might be concentrated on the night side of the moon. We wish to argue that water is actually far more stable on the lunar surface than $\mathrm{SO}_{2}$ or noble gases because of its extremely low vapor pressure at low temperatures and that it may well be present in appreciable quantities in shaded areas in the form of ice.

The pressure of a volatile substance in a closed system is determined by the temperature at the coldest point in the system. The measurements of Pettit and Nicholson [1930], verified by W. M. Sinton (private communication), indicate that the temperature of the lunar surface falls to $120^{\circ} \mathrm{K}$ during the lunar night, and it is reasonable to assume that the temperatures of the permanently shaded areas are equal to or lower than this. It is quite probable that lower temperatures do exist permanently and hence, ice would be even more stable than our calculations indicate. We are using $120^{\circ} \mathrm{K}$, however, because it is a reasonable upper limit. Extrapolation of observed vapor pressure data for ice indicates that at $120^{\circ} \mathrm{K}$ the vapor pressure is about $1.4 \times 10^{-12}$ $\mathrm{mm}$ of mercury. This, then, represents the maximum pressure of water vapor which can exist over the lunar surface, except for brief periods of time, and is many orders of magnitude less

1 Contribution No. 1027, Division of Geological Sciences, California Institute of Technology. than the corresponding pressures of $\mathrm{SO}_{2}$ and the rare gases. For an isothermal atmosphere and constant gas pressure at the surface (corresponding to the vapor pressure) the mass of the atmosphere per unit area of the lunar surface will be independent of both temperature and molecular weight and will correspond at this vapor pressure to $1.1 \times 10^{-11} \mathrm{~g} / \mathrm{cm}^{2}$, or to a total lunar atmospheric water mass of $4.2 \times 10^{\circ}$ grams. These quantities will of course be very sensitive to the assumed temperature of the coldest area. It should be noted that this estimated pressure of water vapor is not inconsistent with the radio occultation measurements of Elsmore [1957].

The least rapid escape rate of water vapor imaginable would be that derived from kinetic theory by assuming direct escape of the fraction of the molecules in the atmosphere which possess velocities greater than the escape velocity. Using Spitzer's [1952] equations and assuming a mean escape temperature of $400^{\circ} \mathrm{K}$ for the sunlit side of the moon, we calculate a relaxation time, $T_{400}=2.5 \times 10^{\mathrm{s}}$ seconds.

On the illuminated side, the water loss would then be $2.2 \times 10^{-20} \mathrm{~g} / \mathrm{cm}_{\mathrm{m}} 2 / \mathrm{sec}$, where $\mathrm{cm}_{\mathrm{m}}{ }^{2}$ refers to the entire lunar surface. Over the life span of the moon $\left(4.56 \times 10^{9}\right.$ years) the loss would be, for unchanging temperature conditions, $3.2 \times 10^{-3} \mathrm{~g} / \mathrm{cm}_{\mathrm{m}}{ }^{2}$.

For a variety of reasons the relaxation time for water in the lunar atmosphere is probably considerably less than that derived above. The temperature of the exosphere may be considerably greater than $400^{\circ} \mathrm{K}$. Other mechanisms may play important roles, for example the one discussed recently by Öpik and Singer [1960]. Öpik and Singer (private communication) in addition have calculated a relaxation time of the order of $10^{6}$ seconds or less for the photodissociation of water. However, it is important to realize that, no matter how short the relaxation time, 
the escape rate cannot exceed the rate of evaporation of ice in the permanently shaded areas.

At $120^{\circ} \mathrm{K}$ the evaporation rate of ice is estimated from kinetic theory to be $3.12 \times 10^{-14}$ $\mathrm{g} / \mathrm{cm}^{2} / \mathrm{sec}$, where the area in this case refers to that of exposed ice. We can estimate an upper limit for the rate of escape of water by assuming that as much as 50 pecent of the lunar surface is ice covered. This estimate gives for the rate of escape $1.6 \times 10^{-14} \mathrm{~g} / \mathrm{cm}_{\mathrm{m}}{ }^{2} / \mathrm{sec}$. Over the life span of the moon the total loss would then be $2.3 \times 10^{3} \mathrm{~g} / \mathrm{cm}_{\mathrm{m}}{ }^{2}$.

If exposed ice exists, its actual area must be much less than 50 per cent of the lunar surface. There appear to be about $6 \times 10^{5}$ craters on the moon with diameters in the range 1 to $5 \mathrm{~km}$ [Kopal, 1960]. About 6 per cent of the moon's surface is covered by these craters. If we assume the crater depth to be $1 / 20$ of the diameter, the permanently shaded areas can occur only north of latitude $78^{\circ} \mathrm{N}$ and south of latitude $78^{\circ} \mathrm{S}$. These areas amount to about 4 per cent of the lunar surface. At a latitude of $84^{\circ}$ the effectively shaded area within a crater appears to be about 5 per cent. Thus, the fraction of the lunar surface which is in permanent shade is estimated roughly to be $0.06 \times 0.04 \times 0.05=1.2 \times 10^{-4}$. If this is true, the area of exposed ice would be $4.5 \times 10^{13}$ $\mathrm{cm}^{2}$ and the escape rate would be $1.9 \times 10^{-18}$ $\mathrm{g} / \mathrm{cm}_{\mathrm{mm}}{ }^{2} / \mathrm{sec}$. Over the life span of the moon this would correspond to a loss of $0.28 \mathrm{~g} / \mathrm{cm}_{\mathrm{m}}{ }^{2}$.

These estimates can be compared with estimates of the quantities of water which would have been liberated from the moon had it evolved as the earth did. We have on the earth today about $1.8 \times 10^{5}$ grams of water per square centimeter of earth surface, corresponding to $1.54 \times 10^{-4}$ grams of water per gram of earth material. If a corresponding amount of water had been liberated on the surface of the moon, it would amount to $3.0 \times 10^{4} \mathrm{~g} / \mathrm{cm}_{\mathrm{m}}{ }^{2}$.

In addition to juvenile water, water is being added sporadically as the result of meteorite impact. Using Brown's [1960] relationships, we estimate that about $10^{8}$ grams of meteoritic matter fall on the moon each year. If we assume a mean water content (including the carbonaceous chondrites) of 0.1 per cent, this corresponds to the addition of $10^{5}$ grams of water each year or $8.3 \times 10^{-21} \mathrm{~g} / \mathrm{cm}_{\mathrm{m}}{ }^{2} / \mathrm{sec}$. A certain fraction of the water in meteorites may rebound with velocities exceeding the escape velocity and hence not be retained. We make no estimate of this escaping fraction. In addition water is liberated from lunar crustal material as the result of impact. If water is liberated suddenly on the lunar surface, whether as the result of meteorite impact or of tectonic activity, the fraction which escapes will depend upon the following relative rates: (a) spread of the gas throughout the atmosphere; $(b)$ condensation of the ice on the night side; $(c)$ escape.

The spread throughout the atmosphere takes place in a time of the order of a day. The relaxation time for condensation on the cold side appears to be of the order of 10 to 15 minutes. Thus, if the water is liberated on the cold side, much of it will be retained during the lunar night and a fraction of it will eventually find its way to the areas of perpetual darkness in the polar regions. If the water is liberated on the hot side, whether or not any is retained will depend upon whether the relaxation time for escape is appreciably longer or shorter than a few hours. Hence, the mass loss of water from the lunar surface may be compensated to some extent by meteorite impact.

In any event, local concentrations of ice on the moon would appear to be well within the realm of possibility. Unfortunately, because ice, if it exists, will be in the dark areas, attempts to determine whether it is present must await the time when suitable instruments can be placed in those areas. When this is done, either a positive or a negative result may reveal a great deal about early lunar history.

Detailed calculations concerning the implications of the existence or nonexistence of ice on the moon are being prepared for publication in this journal in the near future.

Acknowledgment. This work was supported by the National Aeronautics and Space Administration under Grant NSG-56-60.

\section{ReFERENCES}

Brown, Harrison, The density and mass distribution of meteoritic bodies in the neighborhood of the earth's orbit, $J$. Geophys. Research, 65, 1679-1683, 1960.

Elsmore, B., Radio observations of the lunar atmosphere, Phil. Mag., 2, 1040-1046, 1957.

Kopal, Z., Some current problems of lunar topography, Proc. First Intern. Space Sci. Symposium (Kallman, Editor), Interscience Publishers, New York, 1960.

Kuiper, G. P., Planetary atmospheres and their 
origin, in The Atmosphere of the Earth and Planets, Revised Ed., (G. P. Kuiper, Editor), Univ. Chicago Press, 367 pp., 1952.

Öpik, E. J., and S. F. Singer, Escape of gases from the moon, J. Geophys. Research, 65, 3065-3070, 1960.

Pettit, E., and S. B. Nicholson, Lunar radiation and temperatures, Astrophys. $J ., 71,102,1930$.

Spitzer, L., Jr., The terrestrial atmosphere above
$300 \mathrm{~km}$, in The Atmosphere of the Earth and Planets, Revised Ed., (G. P. Kuiper, Editor), Univ. Chicago Press, 239-244, 1952.

Urey, H. C., The Planets, Their Origin and Development, Yale Univ. Press, New Haven, 17-18, 1952.

(Received January 9, 1961; revised February 10, 1961.) 